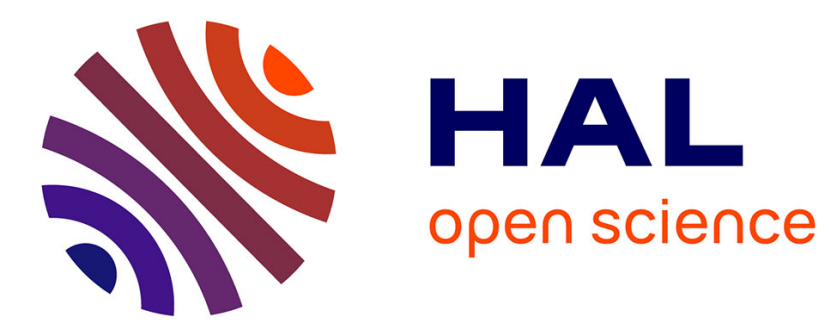

\title{
Polarity mapping of cells and embryos by improved fluorescent solvatochromic pyrene probe
}

Jurga Valanciunaite, Emilie Kempf, Hitomi Seki, Dmytro I Danylchuk, Nadine Peyriéras, Yosuke Niko, Andrey S Klymchenko

\section{To cite this version:}

Jurga Valanciunaite, Emilie Kempf, Hitomi Seki, Dmytro I Danylchuk, Nadine Peyriéras, et al.. Polarity mapping of cells and embryos by improved fluorescent solvatochromic pyrene probe. Analytical Chemistry, 2020, 92 (9), pp.6512. 10.1021/acs.analchem.0c00023 . hal-03030233

\section{HAL Id: hal-03030233 https://hal.science/hal-03030233}

Submitted on 29 Nov 2020

HAL is a multi-disciplinary open access archive for the deposit and dissemination of scientific research documents, whether they are published or not. The documents may come from teaching and research institutions in France or abroad, or from public or private research centers.
L'archive ouverte pluridisciplinaire HAL, est destinée au dépôt et à la diffusion de documents scientifiques de niveau recherche, publiés ou non, émanant des établissements d'enseignement et de recherche français ou étrangers, des laboratoires publics ou privés. 


\title{
Polarity mapping of cells and embryos by improved fluorescent solvatochromic pyrene probe
}

Jurga Valanciunaite, ${ }^{\S, 1}$ Emilie Kempf, ${ }^{\S, 1}$ Hitomi Seki, ${ }^{\S, 2}$ Dmytro I. Danylchuk, ${ }^{1}$ Nadine Peyriéras,${ }^{3}$ Yosuke Niko, ${ }^{2, *}$ Andrey S. Klymchenko ${ }^{1, *}$

${ }^{1}$ Laboratoire de Bioimagerie et Pathologies, UMR 7021 CNRS, Université de Strasbourg, 74 route du Rhin, 67401, Illkirch, France

${ }^{2}$ Research and Education Faculty, Multidisciplinary Science Cluster, Interdisciplinary Science Unit, Kochi University, 2-5-1, Akebono-cho, Kochi-shi, Kochi, 780-8520, Japan.

${ }^{3}$ CNRS USR3695 BioEmergences, Avenue de la Terrasse, 91190 Gif-sur-Yvette, France

*Corresponding authors: andrey.klymchenko@unistra.fr; y.niko@kochi-u.ac.jp

$\S$ These authors contributed equally to this work.

\begin{abstract}
Solvatochromic dyes enable sensing and imaging of biomolecular organization in living systems by monitoring local polarity (lipophilicity), but most such dyes suffer from limited brightness, photostability, lack of a convenient spectral range and limited sensitivity to polarity. Moreover, the presence of an electron acceptor group, typically a carbonyl, in their push-pull structure raises concerns about their potential chemical reactivity within the biological environment. In order to achieve robust bioimaging, we synthesized a push-pull pyrene probe bearing a ketone acceptor group (PK) and compared it with a recently developed aldehyde analogue (PA). We found that in live cells the aldehyde analogue PA transforms slowly (in 100 min) into blue-emissive species, assigned to in situ formation of an imine analogue, whereas the PK probe is stable in the presence of primary amines and inside cells. Like the parent PA, the new probe shows strong solvatochromism and an emission color response to lipid order in membranes (ordered vs. disordered liquid phases), while its blue-shifted absorption is more optimal for excitation with 400-nm light sources. In live cells, the PK probe enables high-contrast polarity mapping of organelles using two-color ratiometric detection, suggesting that polarity increases in the following order: lipid droplets < plasma membranes < endoplasmic reticulum. In the zebrafish embryo, polarity imaging with the PK probe reveals a new dimension in visualizing the organization of tissues - lipophilicity distribution, where biomembranes, lipid droplets, cells, yolk, extracellular space and newly formed organs are revealed by specific emission wavelengths of the probe. The newly developed probe and the proposed approach of polarity mapping open new opportunities for bioimaging at the cellular and animal level.
\end{abstract}




\section{INTRODUCTION}

Dyes that change fluorescence intensity, color and fluorescence lifetime in response to their environment have attracted attention in recent years, due to their potential applications for cellular imaging. ${ }^{1}$ Examples include solvatochromic dyes that are sensitive to solvent polarity, ${ }^{1,2}$ molecular rotors ${ }^{3-5}$ that turn on fluorescence in viscous media and mechano-sensitive (rigidochromic) dyes that change color upon ground-state planarization. ${ }^{6-8}$ Solvatochromic dyes are common in biosensing and bioimaging applications. ${ }^{1}$ These are push-pull fluorophores that undergo an intramolecular excited state charge transfer upon photo-excitation, which increases the dipole moment of their excited state leading to bathochromic (red) shifts of their emission band upon increase in the solvent polarity ${ }^{9,10}$. Solvatochromic dyes can report polarity in different cellular compartments, including cell plasma membranes ${ }^{11,12}$ the endoplasmic reticulum, ${ }^{13,14}$ mitochondria, ${ }^{15}$ the Golgi apparatus, ${ }^{16}$ as well as lipid droplets. ${ }^{14,17,18}$ At the level of lipid membranes, they allow direct monitoring of lipid order in live cells ${ }^{12,14,19-21}$ and can distinguish liquid ordered (Lo) from disordered (Ld) phases, ${ }^{11,22}$ which were hypothesized to play a role in the formation of lipid microdomains (rafts) in cell membranes. ${ }^{23,24}$ In lipid compartments of cell organelles, solvatochromic dyes can detect changes in polarity upon cell starvation and oxidative stress. $^{25,26}$ One should distinguish probes that stain exclusively cell plasma membrane and those that penetrate inside the cells (cell permeant). The former are typically di-4-ANEPPDHQ, ${ }^{27}$ which is a styryl pyridinium derivative, and NR12S ${ }^{12}$ and its recently improved analogues, ${ }^{21}$ based on Nile Red. On the other hand, cell permeant probes are neutral dyes that can rapidly cross cell plasma membranes. Classical examples are Laurdan ${ }^{22,28}$ and Nile Red. ${ }^{29}$ Laurdan is one of the first solvatochromic dyes applied to the studies of lipid rafts in model and cellular membranes. ${ }^{22,28}$ However, limitations are its strong UV absorption and its relatively low photostability. Although Nile Red is better known as a marker of lipid droplets, ${ }^{29}$ recent studies showed its potential for super-resolution imaging of different lipid structures using spectrally resolved imaging. ${ }^{30,31}$ Nile Red operates in a more suitable range of wavelengths than Laurdan, but its photostability and solvatochromism are limited. A number of new solvatochromic dyes were developed to overcome the limitation of these dyes, notably push-pull fluorophores based on anthracene, ${ }^{32}$ fluorene, ${ }^{33}$ pyrene, ${ }^{34}$ isobenzofuran, ${ }^{35}$ DCDHF family, ${ }^{36}$ phenyl-furane, ${ }^{17}$ diphenylamino derivatives, ${ }^{37,38}$ dioxaborine, ${ }^{25}$ etc. Some of these were applied successfully to study lipid droplets $^{14,17,25,37-40}$ and biomembranes. ${ }^{14,25,41,42}$ In particular, we should mention a push-pull pyrene (PA, Figure 1), which, in comparison with Laurdan, shows much brighter fluorescence and better photostability, and absorbs in the convenient violet region, while preserving its sensitivity to lipid order in membranes. ${ }^{14,34}$ Owing to its cell permeability and strong solvatochromism, it enabled mapping polarity in live cells, revealing an increase in polarity from cell plasma membranes towards intracellular membranes. ${ }^{14}$ Moreover, it highlighted the much lower polarity of lipid droplets, in line with several other reports. ${ }^{17,18}$ So far, the PA probe has found applications to study lipid order in membranes of the eye lens, ${ }^{43}$ in sterol trafficking, ${ }^{44}$ and in monitoring the effects of unsaturation in brain phospholipids. ${ }^{42}$

However, an important challenge of push-pull probes is their stability in cellular media against chemical transformations, which is of outmost importance for long-term imaging in cells and small animals. It concerns particularly dyes bearing an aldehyde group, which serves as an electron acceptor in their pushpull system. ${ }^{1}$ The aldehyde group can react with primary amines of proteins and other (bio)molecules, giving rise to the formation of imines (Schiff bases), ${ }^{45,46}$ as recently shown for a push-pull dye aldehyde reacting with an amine-containing binding site of a specially engineered proteins. ${ }^{47}$ Reactivity of 
aldehydes may also contribute to their toxicity. ${ }^{46}$ Moreover, aldehydes can be oxidized to the corresponding acids by aldehyde dehydrogenases. ${ }^{48}$ However, the stability of push-pull dyes bearing aldehyde group has not been characterized in live cells, which raises concerns about their applicability in long-term cell/animal imaging. An attractive alternative as electron acceptor, used for instance in Laurdan, ${ }^{28}$ is a ketone group, which displays similar electron acceptor properties as aldehydes, but much lower reactivity towards amines and oxidative reagents. ${ }^{49}$

In the present work, we synthesized a ketone analogue of PA, push-pull dye 1-acetyl-6-(Ncyclohexyl)aminopyrene (PK, Figure 1) and compared its properties to the parent dye. Our studies in organic solvents showed that PK and PA display almost identical solvatochromism. On the other hand, we found that the PA probe was prone to react with primary amines when bound to lipid membranes, while PK was inert. Long-term experiments in cells revealed that PA faded continuously, with some change in its emission color, whereas PK displayed a stable signal without any change in the emission color. Owing to its stability in cells, the PK probe was applied to image the distribution of polarity in the zebrafish embryo at early and late stages of its development.

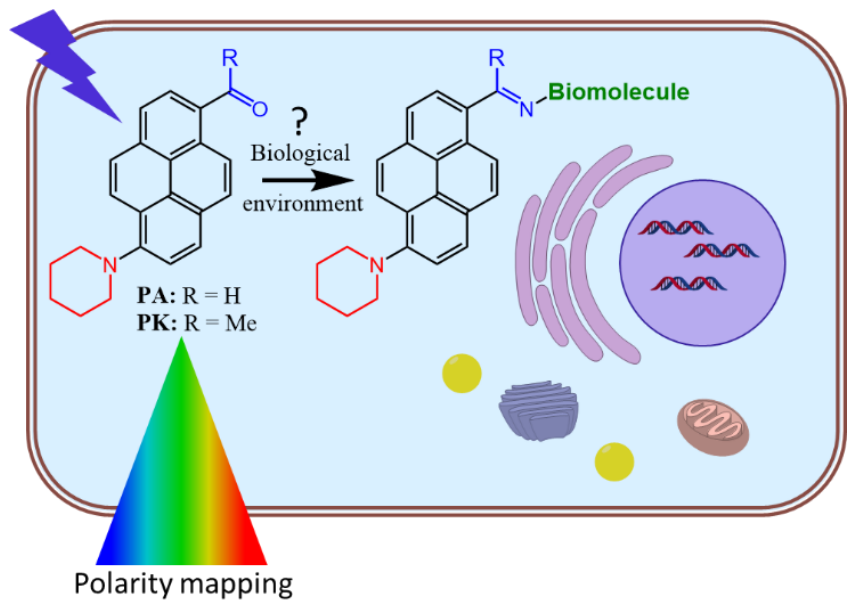

Figure 1. Chemical structures of PA and PK probes in the context of the cells: polarity mapping by solvatochromism and potential reactivity with biomolecules.

\section{EXPERIMENTAL}

\section{Materials}

Synthesis of 1-acetyl-6-(N-cyclohexyl)aminopyrene (PK) is described in SI. Dye, 1-formyl-6-(Ncyclohexyl)aminopyrene (PA), was synthesized as described. ${ }^{34}$ Dioleoylphosphatidylcholine (DOPC), choline (Chol), sphingomyelin (SM) were purchased from Sigma-Aldrich (USA), dodecylamine (DDA) from Alfa Aesar (USA). Nile Red (NR) was purchased from Sigma-Aldrich (USA). Membright 640 was synthesized as described. ${ }^{50}$ MitoTracker ${ }^{\mathrm{TM}}$, Deep Red FM and ER-Tracker ${ }^{\mathrm{TM}}$ Green (BODIPY ${ }^{\circledR}$ FL Glibenclamide) were purchased from Thermo Fisher Scientific (USA). Stock solutions of all probes were prepared in DMSO.

\section{Fluorescence microscopy of cells}


Fluorescence imaging of cells was performed on a Nikon Ti-E inverted microscope (Nikon, Japan) with a 100x objective (Apo TIRF, oil, NA 1.49). RGB imaging of PA and PK was done using a light-emitting diode 395/25 nm (SpectraX, Lumencor) and an RGB camera DS-Fi3 (Nikon, Japan). The camera gain was set to 31.4x for PA and to 14.0x for PK. Exposure time was $500 \mathrm{~ms}$. For ratiometric analysis of images acquired with the RGB camera, the sums of 40 frames in the green and red channels were used. For colocalization studies, two-channel images were acquired sequentially in spinning disk confocal mode (X-light, CrestOptics module) using laser excitation (Oxxius, France). For PK imaging (the green channel), excitation at $405 \mathrm{~nm}$ and an 531/40 nm emission filter were used, except when paired with ERTracker, where the emission filter was $600 / 50 \mathrm{~nm}$. Settings for other probes were: ER-tracker, 488-nm excitation and 531/40-nm emission filter; Nile Red, 488-nm excitation and 600/50-nm emission filter; MitoTracker Deep Red and MemBright 640, 640-nm excitation and 705/72-nm emission filter. For ratiometric imaging in spinning disk mode, 405-nm laser excitation was used, while detection was done using 531/40 nm (Green channel) 600/50-nm (Red channel) emission filters. Images analysis was done using Fiji. ${ }^{51}$

\section{Live zebrafish embryo imaging}

To avoid pigmentation, the nearly transparent Casper line ${ }^{52}$ was used throughout experiments. Embryos were reared and staged as described. ${ }^{53}$ Embryos were collected at the one-cell-stage and raised in embryo medium (EM) solution. 6 and 32 hours post-fertilization embryos were used. For embryo staining, PK stock solution in DMSO $(1 \mathrm{mM})$ was diluted with EM to obtain a $500 \mathrm{nM}$ solution. Embryos were manually dechorionated and kept in $500 \mathrm{nM}$ PK EM solution for $30 \mathrm{~min}$. Prior to imaging, PK-labeled embryos were rinsed two times for five min in EM and kept in EM. Then, embryos were placed in Teflon or $1 \%$ agarose molds depending on the stage. Embryos at $24 \mathrm{hpf}$ were anesthetized in $0.05 \%(\mathrm{w} / \mathrm{v})$ buffered tricaine methane sulfonate (MS222) prior to imaging. All investigations using the zebrafish model were carried out in compliance with the European and international guidelines on animal welfare (Directive 2010/63/EU). Images of zebrafish embryos were acquired on a Zeiss LSM 780 upright confocal microscope using a dipping lens 20x objective (Zeiss W "Plan-Apochromat" 20x/1.0). For excitation a $405 \mathrm{~nm}$ laser was used. Fluorescence was detected in two channels: from 473 to $544 \mathrm{~nm}$ (Green channel) and from 562 to $650 \mathrm{~nm}$ (Red channel).

\section{RESULTS AND DISSCUSION}

In the design of the PK dye, we replaced the aldehyde group with a ketone (acetyl) function, expected to significantly improve its chemical stability in biological environments. On the other hand, the additional ketone group should minimally affect the hydrophobicity of the dye, and therefore should not alter its binding properties to cells, when compared to PA. ${ }^{14}$ The synthesis of PK is described in Supporting Information (Scheme S1). Briefly, the aldehyde group in PA was converted to a secondary alcohol in a Grignard reaction. The resulting alcohol was immediately oxidized to the ketone to afford PK (Figures S1-S3).

The optical properties of PA and PK were compared in solvents of different polarity (Figure 2, Table S1). The absorption and emission maxima of PK were slightly blue-shifted compared to PA in all solvents 
tested (by 9-28 $\mathrm{nm}$ for absorption and by 7-22 $\mathrm{nm}$ for fluorescence). Importantly, the absorption maximum of PK matches well the commonly used light sources around $400 \mathrm{~nm}$ and its cross-talk in absorbance with the $488 \mathrm{~nm}$ laser line is negligible, which are clear advantages compared to the parent PA (Figure 2A). Increase in solvent polarity shifted the emission band of PK to longer wavelengths (119 $\mathrm{nm}$ from heptane to methanol), which indicated strong solvatochromism of the new dye, similarly to PA. In phosphate buffer (PB), the emission maxima of the probes were even more red-shifted, but with band broadening (Figure 2) and drastically reduced quantum yield (QY) (Table S1), indicating quenching in water, typical for solvatochromic dyes. ${ }^{1}$ In other polar and apolar solvents, PA and PK exhibited high QYs close to unity (Table S1).
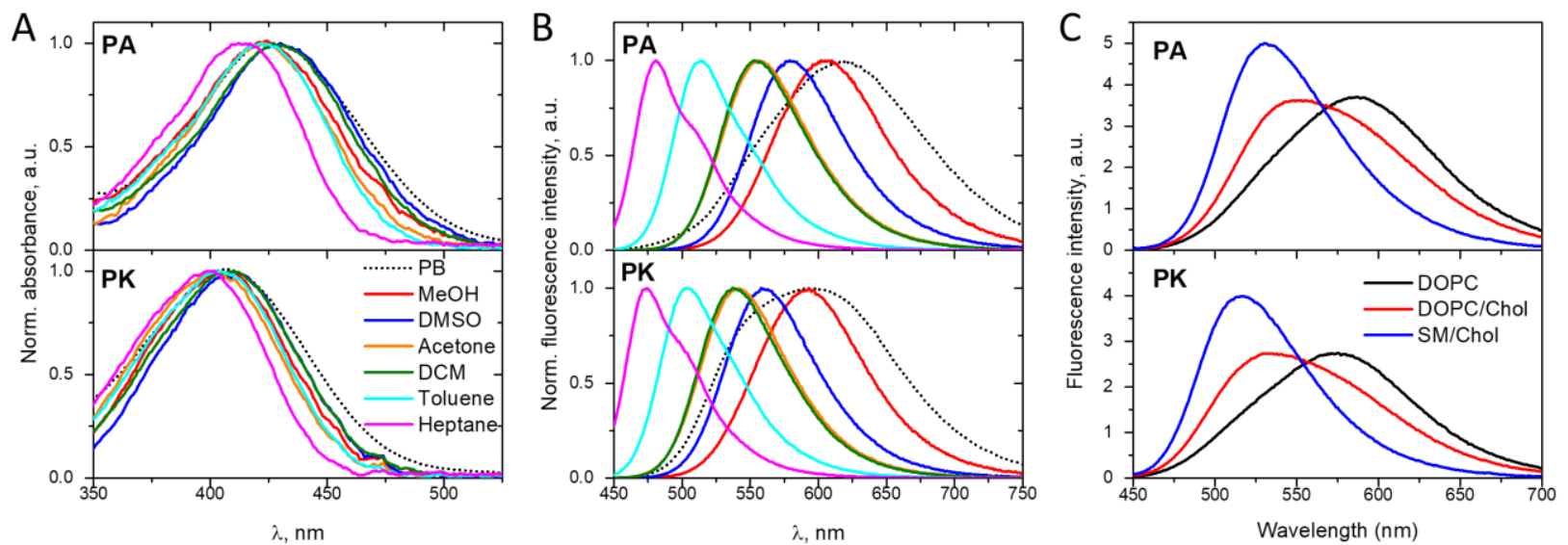

Figure 2. Normalized absorption (A) and emission (B) spectra of PA and PK in solvents of different polarity. (C) Emission spectra of PA and PK in liposomes of different lipid compositions. Probe concentration: $2 \mu \mathrm{M}$, lipid concentration: $200 \mu \mathrm{M}$ (phosphate buffer $20 \mathrm{mM}, \mathrm{pH} 7.4$ ). Excitation wavelength: $430 \mathrm{~nm}$.

To explore the capacity of PK to sense lipid order, we measured emission spectra of PA and PK in large unilamellar vesicles (LUVs) of different lipid composition (Figure 2C, Table S1): (i) dioleoylphosphatidylcholine (DOPC) and DOPC with cholesterol (DOPC/Chol), representing the liquid disordered (Ld) phase, and (ii) sphingomyelin with cholesterol (SM/Chol), representing a liquid ordered (Lo) phase. ${ }^{54,55} \mathrm{PK}$ showed a strong blue shift (by $58 \mathrm{~nm}$ ) of its emission maximum in the Lo phase compared to the Ld phase (composed of DOPC), because of significantly lower polarity and hydration of the Lo phase. ${ }^{11}$ Within the Ld phase, the presence of cholesterol blue-shifted the emission maximum (by $39 \mathrm{~nm}$ ), although the shift was weaker in comparison with that in the Lo phase. Remarkably, the response of PK to differences in lipid compositions was quite similar to that of the parent PA (Figure 2C), while the maximum of the PK emission was systematically blue shifted (by 13-15 nm). Thus, similarly to parent PA, PK is highly sensitive to different lipid phases and to the presence of cholesterol. The spectroscopic response of PK to lipid order and cholesterols is also similar to that of Laurdan. ${ }^{19,28}$ At the same time, PK is clearly advantageous as its absorption ( 400 nm) and emission (500-600 nm) are located in the visible spectral range. We also tested effect of water-soluble proteins and polysaccharides on the PK emission. PK was found insensitive to avidin, lysozyme and alginate, as its fluorescence spectra were close to those in phosphate buffered saline (PBS, Figure S4A, B). A 
fluorescence enhancement and a blue shift of PK were observed in the presence bovine serum albumin (BSA), known for its capacity to bind lipophilic molecules, but these effects were weaker compared to those in lipid vesicles (composed of DOPC/Chol). Importantly, neither BSA nor other tested biomolecules could affect the spectra of PK in LUVs (Figure S4C, D), which indicates that in complex biological environment, $\mathrm{PK}$ is expected to probe lipid membranes with minimal interference of watersoluble proteins and polysaccharides.

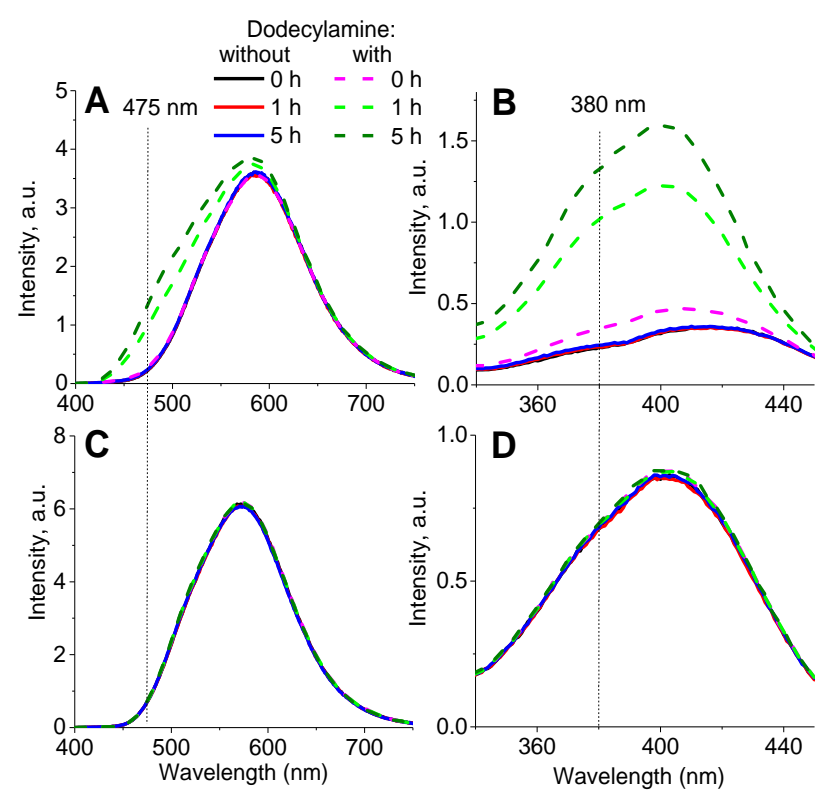

Figure 3. Emission (A, C) and emission excitation (B, D) spectra of PA (A, B) and PK (C, D), respectively, in DOPC liposomes at different incubation time at room temperature without and with dodecylamine. Concentrations of probe, dodecylamine and lipid: 2, 20 and $200 \mu \mathrm{M}$, respectively. Excitation wavelength for (A) and (C): $380 \mathrm{~nm}$; emission wavelength for (B) and (D): $475 \mathrm{~nm}$; they are indicated as dotted lines directly on the corresponding spectra.

To address stability of PA and PK in biological media, we studied the effect of primary amines on the spectroscopic properties of PA and PK (Figure 3). Addition of dodecylamine to PA in DOPC liposomes produced a gradual increase of a short-wavelength shoulder in the emission spectrum at $\sim 470-500 \mathrm{~nm}$, readily detectable already after $1 \mathrm{~h}$ (Figure 3A). In contrast, dodecylamine did not affect the fluorescence spectrum of PK under similar conditions (Figure 3C). Fluorescence excitation spectra of PA, recorded at $475 \mathrm{~nm}$ in the presence of dodecylamine, exhibited an increase in intensity of a short-wavelength shoulder at 370-380 nm (Figure 3B). Again, no changes in the excitation spectra of PK were observed (Figure 3D). The blue-shifted bands in the emission and excitation spectra of PA in the presence of the amine are probably related to formation of a Schiff base (Figure 1). ${ }^{45}$ To identify the adduct, we reacted the PA probe with dodecylamine in ethanol in the presence of acetic acid as catalyst. After a $7 \mathrm{~h}$ reaction, thin layer chromatography showed formation of a new, more hydrophobic product with blue emission, in contrast to PA showing orange emission (Figure S5). Moreover, formation of the imine in the reaction mixture was confirmed by NMR and mass spectrometry (see Supporting Information), although the attempt to purify the product by chromatography resulted in its hydrolysis. Thus, we conclude that the PA probe can be affected by the presence of primary amines, which is not the case for PK. 


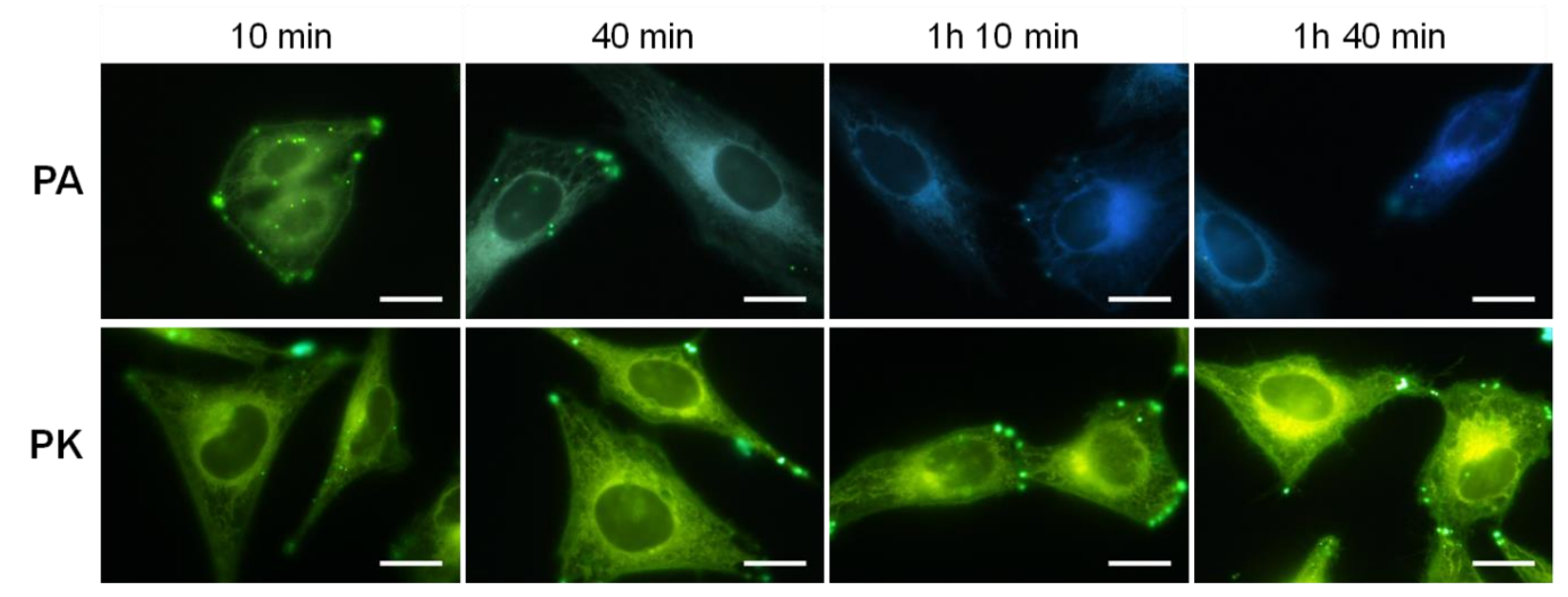

Figure 4. True color images of HeLa cells stained with $10 \mathrm{nM}$ PA and PK at different incubation times taken with RGB camera with an excitation at $395 \mathrm{~nm}$. Scale bar is $20 \mu \mathrm{m}$.

In the next step, we performed fluorescence microscopy of live HeLa cells incubated with PA and PK for different periods of time (Figure 4). Because the PA probe showed changes in its emission spectrum in our model experiments, we used an RGB color camera to verify whether the changes in the true emission color could be observed in live cells also. The RGB color camera detects light in a red, green and blue channel, which corresponds to regions around 480, 530 and $600 \mathrm{~nm}$, respectively. ${ }^{56}$ These three spectral regions adequately cover the emission of the PA and PK dyes, as well as emission of the blueshifted (imine) species of PA observed in the presence of amines (Figure 3). After a 10 min incubation, the fluorescence signal was observed all over the cells. (Figure 4). Initially, we observed a predominant green signal, similar for both probes (Figure 4, first column). However, the difference in the fluorescence color became evident after $40 \mathrm{~min}$ of incubation (Figure 4). Fluorescence of PA inside the cells faded and became blue (Figure 4). In sharp contrast, the green color of cells stained with PK remained the same for all studied incubation times (Figure 4). and the intensity increased, probably because PK continued accumulation in the cells. The observed color change of PA in cells suggests a significant increase in fluorescence centered around 400-500 $\mathrm{nm}$. This observation is in accordance with the results obtained in liposomes with dodecylamine, where the blue-shifted emission increased gradually over time (Figure 3A). Thus, we can conclude that PA undergoes a chemical transformation in cells, probably due to its reaction with primary amines of proteins and other biomolecules. The PK probe did not change its emission color over the time course of the imaging experiments, in line with spectroscopic data in liposomes, suggesting that PK remains stable inside cells.

Since PA was developed primarily for ratiometric imaging of lipid organization in cell membranes, ${ }^{34}$ we checked whether the color change would affect such ratiometric imaging. The intensity ratio of the red to green channel of the RGB camera showed a strong decrease over time for PA but remained stable for PK (Figure S6). However, since the RGB camera is not widely recognized for ratiometric imaging, we performed imaging of cells in green $(<560 \mathrm{~nm})$ and red $(>560 \mathrm{~nm})$ channels using a GEMINI (Hamamatsu) setup that simultaneously splits images into two by using a dichroic mirror. In the case of the PA probe excited at $395 \mathrm{~nm}$, the pseudo-color of cells showed continuous decrease in the Red/Green ratio (Figure S7), which corresponds to the blue shift of the probe observed with the RGB camera (Figure 4) and seen in model experiments in vesicles (Figure 3A). When excited at $470 \mathrm{~nm}$, this change 
in color was no longer observed (Figure S7). Therefore, the newly formed blue-shifted species were not excited at this wavelength, so that only intact PA species were photo-selected. The problem of PA chemical conversion could therefore be partially resolved by using excitation at the red edge. On the other hand, the PK probe, excited at $395 \mathrm{~nm}$, displayed a stable ratiometric signal in cells (Figures 5 and S7). Therefore, PK is clearly preferable over PA for robust ratiometric imaging of cells.

To explore accumulation of PK in different cellular compartments, we studied its co-localization with various markers: plasma membrane (Membright 640 ${ }^{50}$ ); lipid droplets, (Nile Red ${ }^{29}$ ); endoplasmic reticulum (ER-tracker ${ }^{\mathrm{TM}}$ ); and mitochondria (MitoTracker ${ }^{\mathrm{TM}}$ Deep Red) (Figure S8). Plasma membrane marker Membright 640 and PK showed quite different cell staining profiles (Figure S8), suggesting that PK was almost completely internalized. Nevertheless, weak binding of PK to the plasma membrane PK was still observed (arrow; Figure S8). On the other hand, PK showed clear colocalization with Nile Red (Figure S8), a well-known lipophilic probe that distributes over the lipid compartments of the cell, including lipid droplets. ${ }^{29} \mathrm{PK}$ also co-localized with ER-tracker ${ }^{\mathrm{TM}}$, while lipid droplets were stained only by PK (Figure S8). Localization of PK in the ER is not surprising since the ER is the largest continuous intracellular membrane compartment. However, we did not observe clear colocalization for PK and MitoTracker ${ }^{\mathrm{TM}}$ Deep Red, probably because our dye is a neutral molecule, and, unlike the cationic dyes, does not accumulate well in mitochondria. ${ }^{57} \mathrm{PK}$ showed colocalization with most of organelle trackers (except mitochondria), probably because it distributes over different lipid compartments in these organelles.

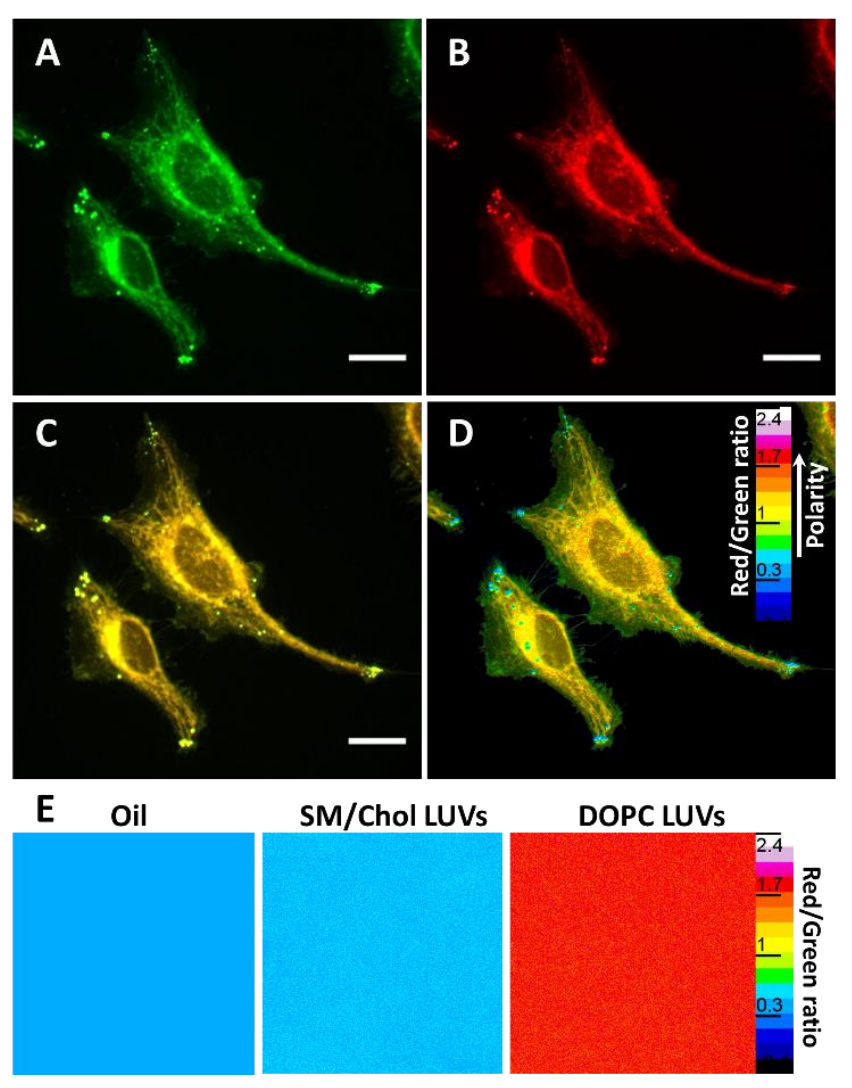

Figure 5. Fluorescence images of HeLa cells stained with $20 \mathrm{nM}$ PK and measured at two channels in spinning disk mode: $\mathrm{A}-$ green channel $\left(\lambda_{\mathrm{ex}}=405 \mathrm{~nm}, \lambda_{\mathrm{em}}=531 / 40 \mathrm{~nm}\right), \mathrm{B}-$ red channel $\left(\lambda_{\mathrm{ex}}=405 \mathrm{~nm}\right.$, $\left.\lambda_{\mathrm{em}}=600 / 50 \mathrm{~nm}\right), \mathrm{C}-$ merged image of green and red channels, $\mathrm{D}-\mathrm{Red} / \mathrm{Green}$ ratio image with a pseudo- 
color scale reflecting probe environmental polarity. E- calibration ratiometric images for PK probe in environments of different polarity: Labrafac oil, SM/Chol vesicles (LUVs) and DOPC LUVs. Polarity increases from blue to red pseudo-color. Scale bar is $20 \mu \mathrm{m}$.

Owing to sensitivity of PK to environment polarity and lipid order, we applied it to map polarity distributions in cells by recording confocal (spinning-disk) images in two colors, green (531/40 nm filter) and red (600/50 $\mathrm{nm}$ filter) and further merging them or making a Red/Green ratiometric image (Figure 5). The PK probe showed remarkable heterogeneity in its emission color, i.e. uneven polarity distribution in lipid compartments of the cell (Figure 5 and Figure S7). This is in line with earlier studies using PA. ${ }^{14}$ To mimic polarity of cellular lipid structures, we tested several model systems: (i) labrafac oil, a low polarity medium similar to the oily core of lipid droplets; (ii) lipid vesicles composed of sphingomyelin and cholestrerol (SM/Chol), representing the Lo phase of membranes and (iii) lipid vesicles composed of unsaturated lipid DOPC, which corresponds to the Ld phase. ${ }^{54,55}$ In these model samples, the $\mathrm{Red} / \mathrm{Green}$ ratio increased in the following order: labrafac $<\mathrm{SM} / \mathrm{Chol}<\mathrm{DOPC}$, corresponding to a color change from blue to light blue and further to red (Figure 5E), which reflects the growth in the environment polarity. Indeed, while labrafac oil and membranes in the Lo phase are rather apolar, bilayers of the Ld phase are significantly more polar and hydrated. ${ }^{11}$ In cells, the lowest value for the Red/Green ratio (corresponding to blue pseudo-color in the ratio and green in the merged images, Figure 5) was observed in the form of dots that can be readily assigned to lipid droplets, in line with our colocalization studies. This spectral response of the PK probe, close to that seen in labrafac oil, was expected, because lipid droplets are the compartments of the lowest polarity in cells. ${ }^{14,17,18}$ Relatively low polarity was observed for plasma membranes that appeared at the cell contour (green in ratiometric and green-yellow in the merged images). The polarity was significantly higher for continuous structures inside the cells, which appeared in colors ranging from green-yellow to orange. According to our colocalization studies, these structures could be assigned to intracellular membranes, mainly the endoplasmic reticulum. When compared to our data in lipid vesicles, plasma membranes appeared closer to the low polar Lo phase, whereas intracellular membranes matched better to much more polar Ld phase. This corroborates previous reports that use $\mathrm{PA}^{14}$ and Laurdan ${ }^{58}$ probes and is related to the much lower concentration of cholesterol and SM in the intracellular membranes (close to the Ld phase) compared to plasma membranes (matching the Lo phase). ${ }^{14,59}$ Remarkably, intracellular membranes exhibited an apparent gradual change of color from green at the cell extremities to yellow, closer to the nucleus (higher polarity). We can attribute that to gradients of cholesterol inside the cells due to the activity of cholesterol transporters ${ }^{60}$ as well as to presence of different intracellular organelles with distinct lipid order and cholesterol content. ${ }^{59}$ Nevertheless, one cannot exclude that this apparent polarity gradient could be related to uneven distribution of ER in cells and contribution to the signal from plasma and nuclear membranes. 


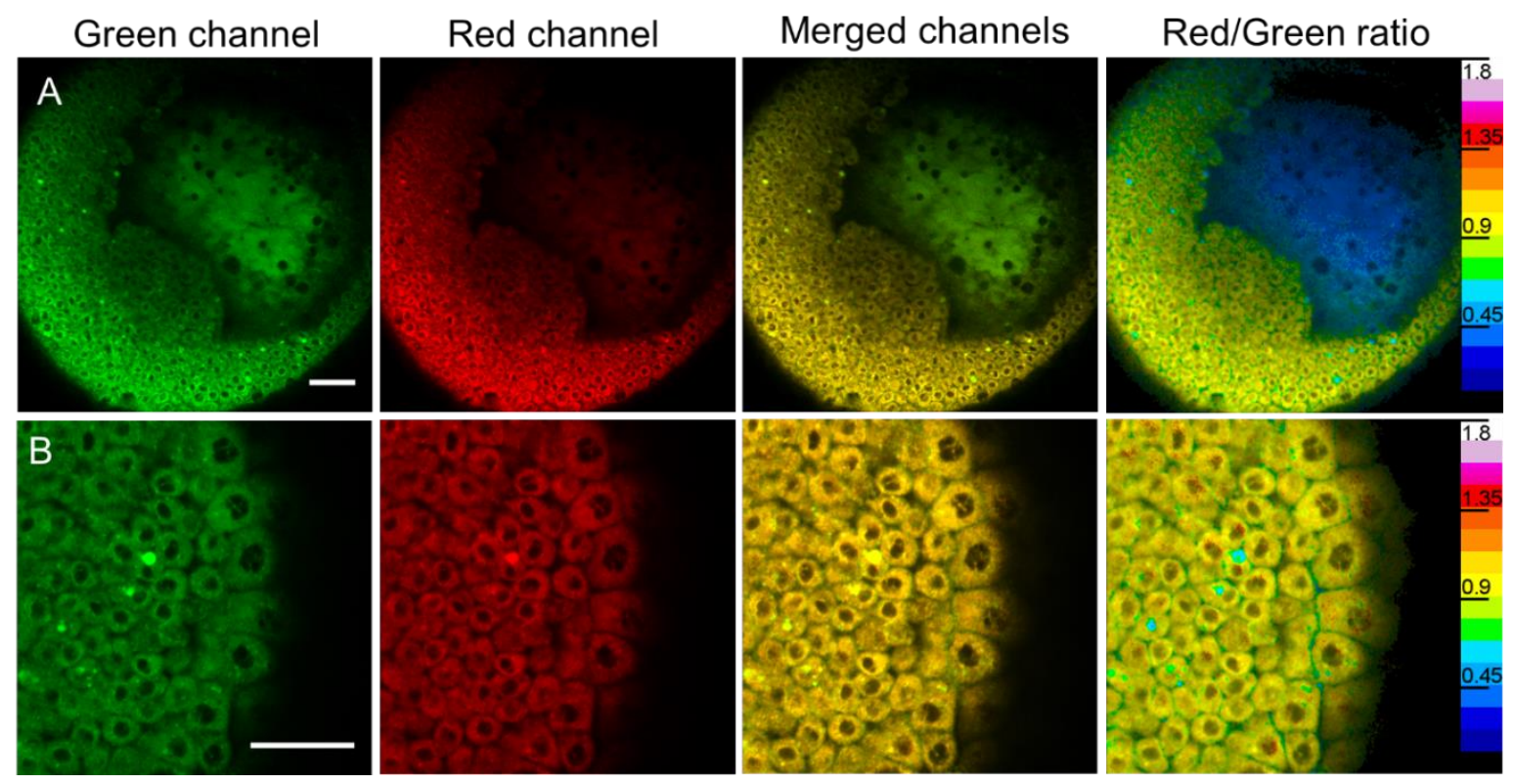

Figure 6. Two-color and ratiometric confocal imaging of an early zebrafish embryo (6h postfertilization) stained with $500 \mathrm{nM}$ of PK probe, using 1.1 (A) and 2.5 (B) zoom (Objective 20x). Excitation wavelength was $405 \mathrm{~nm}$. Emission was collected at two channels: 473-544 nm (green channel) and $562-650 \mathrm{~nm}$ (red channel). Scale bar is $50 \mu \mathrm{m}$.

Finally, we evaluated whether the PK probe can be used to stain and image polarity distributions in small animals. To this end we used the zebrafish embryo, which is a perfect animal model due it its optical transparency. ${ }^{53,61,62}$ First, we imaged an early embryo (6h post-fertilization) stained with PK using twocolor confocal microscopy. The embryo stained with PK showed strong signal in both green and red channels, providing high color contrast in the merged images (Figure 6). The control experiments without the dye showed practically zero signal in both detection channels (Figure S9), indicating a negligible effect of auto-fluorescence in our imaging conditions. We found that the yolk part showed the lowest polarity within the embryo (blue pseudo-color in the Red/Green ratio image, Figure 6A), probably because it is rich in lipids and lipoproteins. ${ }^{63}$ In zoomed images of the embryo (Figure 6B), we observed that cell borders, probably plasma membranes, appeared in green, while the intracellular content appeared in orange. These results are fully in line with in vitro studies showing that intracellular membranes exhibit higher polarity than the plasma membranes (Figure 5). However, in this experiment we can now observe this phenomenon directly in the cells of a live embryo. We also observed some bluegreen dots of different sizes in these images, which we assume to correspond to low polar lipid droplets. 

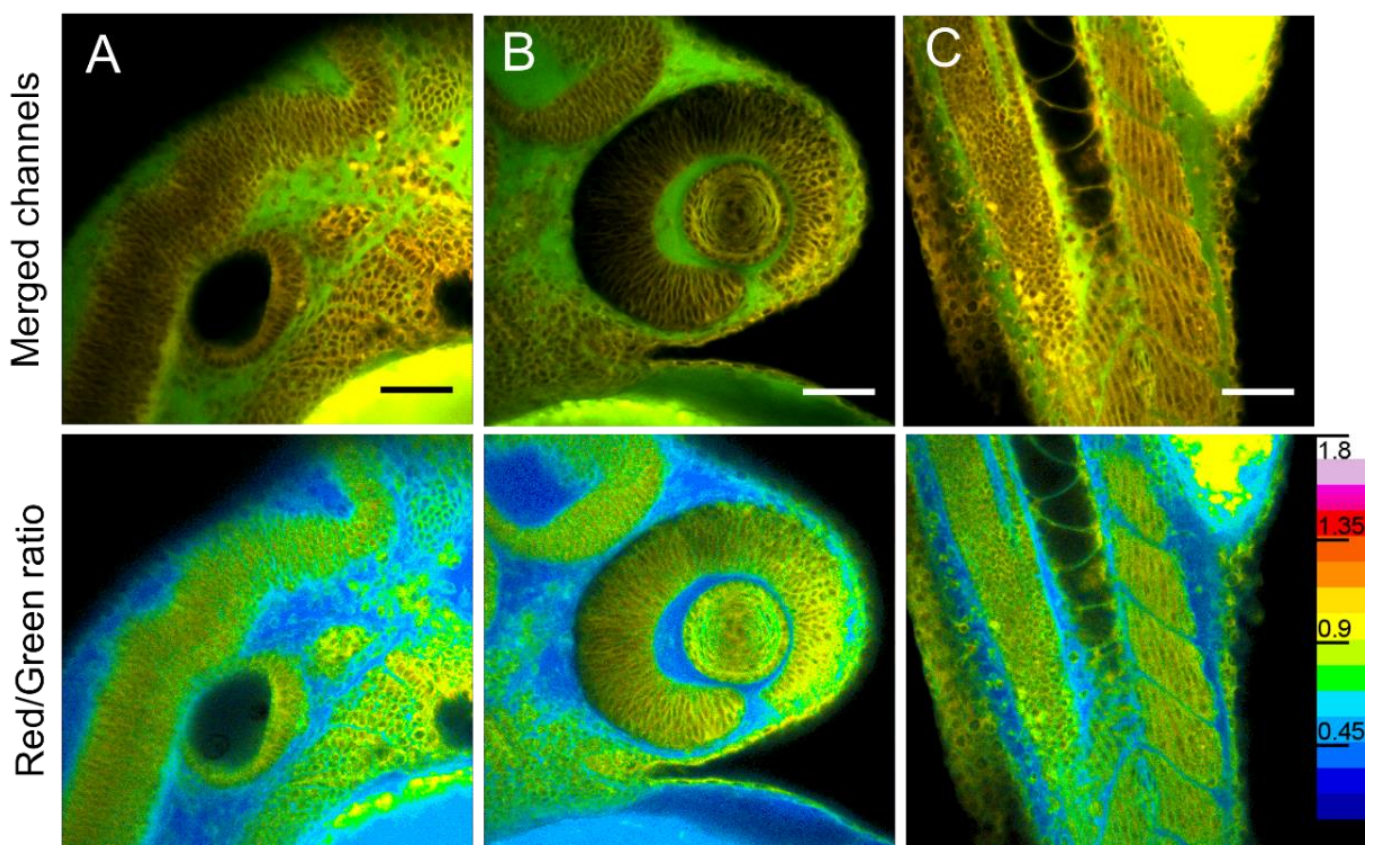

Figure 7. Two-color and ratiometric confocal imaging of different parts of a zebrafish embryo (32 $\mathrm{h}$ post-fertilization) stained with $500 \mathrm{nM}$ of PK probe: ear (A), eye (B) and tail (C). Excitation wavelength was $405 \mathrm{~nm}$. Emission was collected at two channels: GFP (green) and mCherry (red) channels. Scale bar is $50 \mu \mathrm{m}$.

We performed similar imaging experiments on a late zebrafish embryo ( $32 \mathrm{~h}$ post-fertilization), which gave striking two-color images (Figure 7, Movies S1-S3), whereas the control embryos without the dye displayed nearly zero auto-fluorescence (Figure S10). Both merged and ratio images showed dramatic heterogeneity in polarity distribution inside the ear, eye and tail of the embryo (Figure 7), revealing fine morphological details. In the Red/Green ratio images, the extracellular spaces located between the epithelial tissues appeared in blue pseudo-color, suggesting their lowest polarity, similar to that of the yolk. ${ }^{64}$ Some of these regions could be assigned to yolk syncytial layers, ${ }^{64}$ while the others more distant from yolk are probably loose arrays of cells forming new tissues. Blue pseudo-color of PK suggests that these regions are filled with low polar yolk-like liquid, probably rich in nutrients. Some low polarity regions could be also assigned to the ventricular space, which is also known to contain lipid particles. ${ }^{65}$ The separation between cells (generally plasma membranes) appeared in green (intermediate polarity), whereas the intracellular compartments appeared as the most polar regions, in line with the data on the HeLa cell line (Figure 5) and on the early embryo (Figure 6). Although detailed understanding of the observed remarkable heterogeneity in the embryo is beyond the scope of this work, our results show the great potential of polarity mapping in small animals, using PK as a solvatochromic dye.

\section{Conclusions}

Polarity mapping using solvatochromic probes is an emerging sensing and imaging modality at the cellular and animal level. It requires robust probes that feature high brightness, solvatochromism and chemical stability over long incubation periods. A number of solvatochromic probes, including the 
recently developed push-pull pyrene PA, bear an aldehyde electron acceptor group, which may pose a problem because of its potential reactivity. To overcome this problem, we synthesized an improved analogue, PK, in which the aldehyde group is replaced by a ketone (acetyl). PK showed advantageous spectroscopic properties, as its absorption maximum matched well to common violet light sources around $400 \mathrm{~nm}$ and did not cross-talk with the blue laser at $488 \mathrm{~nm}$, commonly used for green makers (GFP, FITC, etc). Moreover, the PK dye preserved its sensitivity to lipid order, by changing its emission color between liquid ordered and liquid disordered phases. Experiments in model membranes revealed that the aldehyde analogue PA underwent an in situ reaction with primary amines, yielding a blue-shifted emissive species, whereas the ketone derivative PK remained stable. The spectral changes of PA are related to transformation of aldehyde into an imine, with weaker electron accepting properties. PA and PK also showed very different stability profiles in live cells: PA progressively changed its emission color from green to blue, whereas PK exhibited a stable emission color over time. These results suggest that replacing the aldehyde with a ketone in the push-pull pyrene allows us to drastically improve chemical stability of the probe in biological environments. Importantly, the PK probe enabled high-contrast polarity mapping of organelles in live cells using two-color ratiometric detection, suggesting that polarity increases in the following order: lipid droplets < plasma membranes < endoplasmic reticulum. This rank order reflects the uneven distribution and organization of lipids in cells. In the zebrafish embryo, ratiometric two-color imaging with the PK probe revealed remarkable uneven distribution in the environment polarity, where plasma and intracellular membranes, lipid droplets, yolk, extracellular space, including yolk syncytial layers, and newly formed organs can be distinguished by a characteristic emission color of the probe. We found that the most apolar regions of zebrafish embryo are yolk and extracellular space with newly forming organs, filled with lipidic nutrients. The polarity of lipid compartments in the embryo gradually increased towards cell plasma membranes reaching maximal values at the level of intracellular membranes. Overall, the polarity mapping with the developed PK probe constitutes a new dimension in bioimaging at the cellular and organismal level.

\section{Supporting information}

The Supporting Information is available free of charge at the ACS Publication website. It describes the synthesis of PK, experimental spectroscopy and cell preparation details, and additional experimental data.

\section{Acknowledgements}

This work was supported by the European Research Council ERC Consolidator grant BrightSens 648528. J. Vermot is acknowledged for fruitful discussions. We thank BioEmergences platform and Manon Mehraz (USR3695 CNRS, Gif-sur-Yvette, France) for zebrafish imaging, supported by France BioImaging ANR-10-INBS-04 and Morphoscope ANR11-EQPX-0029. 


\section{References}

(1) Klymchenko, A. S. Acc. Chem. Res. 2017, 50, 366-375.

(2) Reichardt, C. Chem. Rev. 1994, 94, 2319-2358.

(3) Kuimova, M. K.; Yahioglu, G.; Levitt, J. A.; Suhling, K. J. Am. Chem. Soc. 2008, 130, 6672-6673.

(4) Kuimova, M. K. Phys. Chem. Chem. Phys. 2012, 14, 12671-12686.

(5) Haidekker, M. A.; Theodorakis, E. A. Org. Biomol. Chem. 2007, 5, 1669-1678.

(6) Colom, A.; Derivery, E.; Soleimanpour, S.; Tomba, C.; Dal Molin, M.; Sakai, N.; Gonzalez-Gaitan, M.; Matile, S.; Roux, A. Nat. Chem. 2018, 10, 1118-1125.

(7) Dal Molin, M.; Verolet, Q.; Colom, A.; Letrun, R.; Derivery, E.; Gonzalez-Gaitan, M.; Vauthey, E.; Roux, A.; Sakai, N.; Matile, S. J. Am. Chem. Soc. 2015, 137, 568-571.

(8) Goujon, A.; Colom, A.; Strakova, K.; Mercier, V.; Mahecic, D.; Manley, S.; Sakai, N.; Roux, A.; Matile, S. J. Am. Chem. Soc. 2019, 141, 3380-3384.

(9) Lippert Von, E. Z. Electrochem. 1957, 61, 962-975.

(10) Mataga, N.; Kaifu, Y.; Koizumi, M. Bull. Chem. Soc. Jpn. 1956, 29, 465-470.

(11) Klymchenko, A. S.; Kreder, R. Chem. Biol. 2014, 21, 97-113.

(12) Kucherak, O. A.; Oncul, S.; Darwich, Z.; Yushchenko, D. A.; Arntz, Y.; Didier, P.; Mely, Y.; Klymchenko, A. S. J. Am. Chem. Soc. 2010, 132, 4907-4916.

(13) Yang, Z.; He, Y.; Lee, J. H.; Chae, W. S.; Ren, W. X.; Lee, J. H.; Kang, C.; Kim, J. S. Chem. Commun. 2014, 50, 11672-11675.

(14) Niko, Y.; Didier, P.; Mely, Y.; Konishi, G.; Klymchenko, A. S. Sci. Rep. 2016, 6, 18870.

(15) Jiang, N.; Fan, J. L.; Xu, F.; Peng, X. J.; Mu, H. Y.; Wang, J. Y.; Xiong, X. Q. Angew. Chem. Int. Ed. Engl. 2015, $54,2510-2514$.

(16) Li, P.; Guo, X. M.; Bai, X. Y.; Wang, X.; Ding, Q.; Zhang, W.; Zhang, W.; Tang, B. Anal. Chem. 2019, 91, 33823388.

(17) Collot, M.; Bou, S.; Fam, T. K.; Richert, L.; Mély, Y.; Danglot, L.; Klymchenko, A. S. Anal. Chem. 2018, 91, 1928-1935.

(18) Ghosh, C.; Nandi, S.; Bhattacharyya, K. Chem. Phys. Lett. 2017, 670, 27-31.

(19) Owen, D. M.; Rentero, C.; Magenau, A.; Abu-Siniyeh, A.; Gaus, K. Nat. Protoc. 2012, 7, 24-35.

(20) Sezgin, E.; Schneider, F.; Zilles, V.; Urbancic, I.; Garcia, E.; Waithe, D.; Klymchenko, A. S.; Eggeling, C.

Biophys. J. 2017, 113, 1321-1330.

(21) Danylchuk, D. I.; Moon, S.; Xu, K.; Klymchenko, A. S. Angew. Chem. Int. Ed. Engl. 2019, 58, 14920-14924.

(22) Bagatolli, L. A. Biochim. Biophys. Acta Biomembr. 2006, 1758, 1541-1556.

(23) Lingwood, D.; Simons, K. Science 2010, 327, 46-50.

(24) Sezgin, E.; Levental, I.; Mayor, S.; Eggeling, C. Nat. Rev. Mol. Cell Biol. 2017, 18, 361.

(25) Ashoka, A. H.; Ashokkumar, P.; Kovtun, Y. P.; Klymchenko, A. S. J. Phys. Chem. Lett. 2019, 10, 2414-2421.

(26) Xiao, H. B.; Wu, C. C.; Li, P.; Bo, T. G. Anal. Chem. 2018, 90, 6081-6088.

(27) Jin, L.; Millard, A. C.; Wuskell, J. P.; Dong, X. M.; Wu, D. Q.; Clark, H. A.; Loew, L. M. Biophys. J. 2006, 90, 2563-2575.

(28) Parasassi, T.; Krasnowska, E. K.; Bagatolli, L.; Gratton, E. J. Fluoresc. 1998, 8, 365-373.

(29) Greenspan, P.; Mayer, E. P.; Fowler, S. D. The Journal of Cell Biology 1985, 100, 965.

(30) Moon, S.; Yan, R.; Kenny, S. J.; Shyu, Y.; Xiang, L. M.; Li, W.; Xu, K. J. Am. Chem. Soc. 2017, 139, 1094410947.

(31) Bongiovanni, M. N.; Godet, J.; Horrocks, M. H.; Tosatto, L.; Carr, A. R.; Wirthensohn, D. C.; Ranasinghe, R. T.; Lee, J. E.; Ponjavic, A.; Fritz, J. V.; Dobson, C. M.; Klenerman, D.; Lee, S. F. Nat. Commun. 2016, 7, 9.

(32) Lu, Z.; Lord, S. J.; Wang, H.; Moerner, W. E.; Twieg, R. J. J. Org. Chem. 2006, 71, 9651-9657.

(33) Kucherak, O. A.; Didier, P.; Mely, Y.; Klymchenko, A. S. J. Phys. Chem. Lett. 2010, 1, 616-620.

(34) Niko, Y.; Kawauchi, S.; Konishi, G. Chem-Eur J 2013, 19, 9760-9765.

(35) Norris, S. R.; Warner, C. C.; Lampkin, B. J.; Bouc, P.; VanVeller, B. Org. Lett. 2019, 21, 3817-3821. 
(36) Lord, S. J.; Conley, N. R.; Lee, H. L. D.; Nishimura, S. Y.; Pomerantz, A. K.; Willets, K. A.; Lu, Z. K.; Wang, H.; Liu, N.; Samuel, R.; Weber, R.; Semyonov, A.; He, M.; Twieg, R. J.; Moerner, W. E. ChemPhysChem 2009, 10, 5565.

(37) Jiang, M. J.; Gu, X. G.; Lam, J. W. Y.; Zhang, Y. L.; Kwok, R. T. K.; Wong, K. S.; Tang, B. Z. Chem. Sci. 2017, 8, 5440-5446.

(38) Zheng, Z.; Zhang, T. F.; Liu, H. X.; Chen, Y. C.; Kwok, R. T. K.; Ma, C.; Zhang, P. F.; Sung, H. H. Y.; Williams, I. D.; Lam, J. W. Y.; Wong, K. S.; Tang, B. Z. ACS Nano 2018, 12, 8145-8159.

(39) Yin, J. L.; Peng, M.; Ma, Y. Y.; Guo, R.; Lin, W. Y. Chem. Commun. 2018, 54, 12093-12096.

(40) Fam, T. K.; Klymchenko, A. S.; Collot, M. Materials 2018, 11, 1768.

(41) Shaya, J.; Collot, M.; Benailly, F.; Mahmoud, N.; Mely, Y.; Michel, B. Y.; Klymchenko, A. S.; Burger, A. ACS Chem. Biol. 2017, 12, 3022-3030.

(42) Manni, M. M.; Tiberti, M. L.; Pagnotta, S.; Barelli, H.; Gautier, R.; Antonny, B. eLife 2018, 7, e34394.

(43) Sherin, P. S.; Lopez-Duarte, I.; Dent, M. R.; Kubankova, M.; Vysniauskas, A.; Bull, J. A.; Reshetnikova, E. S.; Klymchenko, A. S.; Tsentalovich, Y. P.; Kuimova, M. K. Chem. Sci. 2017, 8, 3523-3528.

(44) Mesmin, B.; Bigay, J.; Polidori, J.; Jamecna, D.; Lacas-Gervais, S.; Antonny, B. Embo J. 2017, 36, 3156-3174.

(45) Layer, R. W. Chem. Rev. 1963, 63, 489-510.

(46) LoPachin, R. M.; Gavin, T. Chem. Res. Toxicol. 2014, 27, 1081-1091.

(47) Sheng, W.; Nick, S. T.; Santos, E. M.; Ding, X.; Zhang, J.; Vasileiou, C.; Geiger, J. H.; Borhan, B. Angew. Chem. Int. Ed. Engl. 2018, 57, 16083-16087.

(48) Singh, S.; Brocker, C.; Koppaka, V.; Chen, Y.; Jackson, B. C.; Matsumoto, A.; Thompson, D. C.; Vasiliou, V. Free Radic. Biol. Med. 2013, 56, 89-101.

(49) Knipe, A. C. In Organic Reaction Mechanisms · 2008; John Wiley \& Sons Ltd, 2011.

(50) Collot, M.; Ashokkumar, P.; Anton, H.; Boutant, E.; Faklaris, O.; Galli, T.; Mely, Y.; Danglot, L.; Klymchenko, A. S. Cell Chem. Biol. 2019, 26, 600-614.e607.

(51) Schindelin, J.; Arganda-Carreras, I.; Frise, E.; Kaynig, V.; Longair, M.; Pietzsch, T.; Preibisch, S.; Rueden, C.; Saalfeld, S.; Schmid, B.; Tinevez, J. Y.; White, D. J.; Hartenstein, V.; Eliceiri, K.; Tomancak, P.; Cardona, A. Nat Methods 2012, 9, 676-682.

(52) White, R. M.; Sessa, A.; Burke, C.; Bowman, T.; LeBlanc, J.; Ceol, C.; Bourque, C.; Dovey, M.; Goessling, W.; Burns, C. E.; Zon, L. I. Cell Stem Cell 2008, 2, 183-189.

(53) Adjili, S.; Favier, A.; Fargier, G.; Thomas, A.; Massin, J.; Monier, K.; Favard, C.; Vanbelle, C.; Bruneau, S.;

Peyrieras, N.; Andraud, C.; Muriaux, D.; Charreyre, M. T. Biomaterials 2015, 46, 70-81.

(54) Veatch, S. L.; Keller, S. L. Biophys. J. 2003, 85, 3074-3083.

(55) Sankaram, M. B.; Thompson, T. E. Biochemistry 1990, 29, 10670-10675.

(56) Wang, X. D.; Gorris, H. H.; Stolwijk, J. A.; Meier, R. J.; Groegel, D. B. M.; Wegener, J.; Wolfbeis, O. S. Chem.

Sci. 2011, 2, 901-906.

(57) Zhu, H.; Fan, J. L.; Du, J. J.; Peng, X. J. Acc. Chem. Res. 2016, 49, 2115-2126.

(58) Golfetto, O.; Hinde, E.; Gratton, E. Biophys. J. 2013, 104, 1238-1247.

(59) Mesmin, B.; Maxfield, F. R. Biochim. Biophys. Acta Mol. Cell. Biol. Lipids 2009, 1791, 636-645.

(60) von Filseck, J. M.; Vanni, S.; Mesmin, B.; Antonny, B.; Drin, G. Nat. Commun. 2015, 6, 6671.

(61) Evensen, L.; Johansen, P. L.; Koster, G.; Zhu, K.; Herfindal, L.; Speth, M.; Fenaroli, F.; Hildahl, J.; Bagherifam, S.; Tulotta, C.; Prasmickaite, L.; Maelandsmo, G. M.; Snaar-Jagalska, E.; Griffiths, G. Nanoscale 2016, 8, 862-877. (62) Andreiuk, B.; Reisch, A.; Lindecker, M.; Follain, G.; Peyrieras, N.; Goetz, J. G.; Klymchenko, A. S. Small 2017, $13,13$.

(63) Miyares, R. L.; de Rezende, V. B.; Farber, S. A. Dis. Model Mech. 2014, 7, 915-927.

(64) Carvalho, L.; Heisenberg, C. P. Trends Cell Biol. 2010, 20, 586-592.

(65) Feliciano, D. M.; Zhang, S. L.; Nasrallah, C. M.; Lisgo, S. N.; Bordey, A. Plos One 2014, 9, e88810. 
For Table of Contents Only

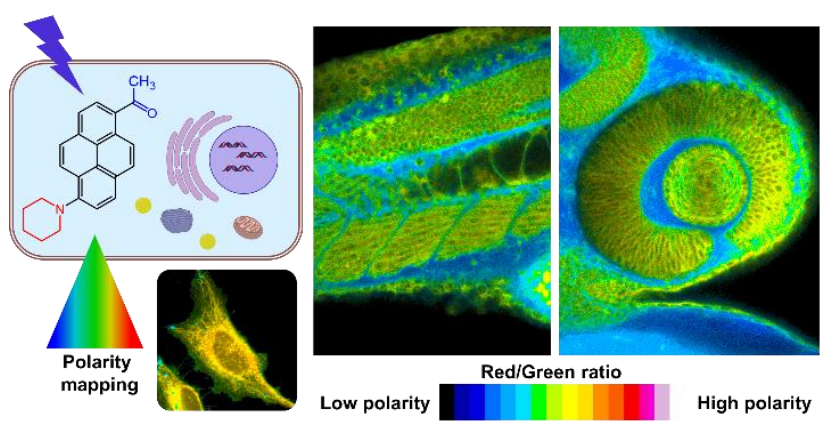

